Published in final edited form as:

J Am Acad Orthop Surg. 2009 June ; 17(6): 389-396.

\title{
Diagnosis of Carpal Tunnel Syndrome
}

\section{Dr. Michael Warren Keith, MD [Professor],}

Orthopaedics and Biomedical Engineering, and Chief, Hand Surgery Service, MetroHealth Medical Center, Case Western Reserve University, Cleveland, $\mathrm{OH}$

\section{Dr. Victoria Masear, MD [Orthopaedic Surgeon],}

Orthopaedic Specialists of Alabama, Birmingham, AL

\section{Dr. Kevin Chung, MD, MS [Professor],}

Plastic Surgery, University of Michigan Department of Surgery, University of Michigan Medical Center, Ann Arbor, MI

Dr. Kent Maupin, MD [Orthopaedic Surgeon], Michigan Hand Center, Grand Rapids, MI

Dr. Michael Andary, MD, MS [Professor],

Department of Physical Medicine and Rehabilitation, Michigan State University, East Lansing, MI

Dr. Peter C. Amadio, MD [Orthopaedic Surgeon],

Mayo Clinic, and Professor of Orthopedics, Mayo Clinic College of Medicine, Rochester, MN

\section{Dr. Richard W. Barth, MD [Chief],}

Section of Orthopaedic Surgery, and Chief, Section of Hand Surgery, Sibley Memorial Hospital, Washington, DC, and a member of the Board of Councilors, American Academy of Orthopaedic Surgeons, Rosemont, IL

Dr. William C. Watters III, MD [Orthopaedic Surgeon],

Bone and Joint Clinic of Houston, Houston, TX

Dr. Michael J. Goldberg, MD [Chief],

Skeletal Dysplasia Clinic, Seattle Children's Hospital, Seattle, WA

Dr. Robert H. Haralson III, MD, MBA [Executive Director of Medical Affairs],

American Academy of Orthopaedic Surgeons

\section{Dr. Charles M. Turkelson, PhD [Director], and}

Department of Research and Scientific Affairs, American Academy of Orthopaedic Surgeons

\section{Dr. Janet L. Wies, MPH [Manager]}

Clinical Practice Guidelines Unit, American Academy of Orthopaedic Surgeons

\section{Abstract}

This clinical practice guideline was created to improve patient care by outlining the appropriate information-gathering and decision-making processes involved in managing the diagnosis of 
carpal tunnel syndrome. The methods used to develop this clinical practice guideline were designed to combat bias, enhance transparency, and promote reproducibility. The guideline's recommendations are as follows: The physician should obtain an accurate patient history. The physician should perform a physical examination of the patient that may include personal characteristics as well as performing a sensory examination, manual muscle testing of the upper extremity, and provocative and/or discriminatory tests for alternative diagnoses. The physician may obtain electrodiagnostic tests to differentiate among diagnoses. This may be done in the presence of thenar atrophy and/or persistent numbness. The physician should obtain electrodiagnostic tests when clinical and/or provocative tests are positive and surgical management is being considered. If the physician orders electrodiagnostic tests, the testing protocol should follow the American Academy of Neurology/American Association of Neuromuscular and Electrodiagnostic Medicine/American Academy of Physical Medicine and Rehabilitation guidelines for diagnosis of carpal tunnel syndrome. In addition, the physician should not routinely evaluate patients suspected of having carpal tunnel syndrome with new technology, such as magnetic resonance imaging, computed tomography, and pressure-specified sensorimotor devices in the wrist and hand. This decision was based on an additional nonsystematic literature review following the face-to-face meeting of the work group.

\section{Overview and Rationale}

This clinical practice guideline was approved by the American Academy of Orthopaedic Surgeons (AAOS) in May 2007. The AAOS created this clinical practice guideline to improve patient care by outlining the appropriate information-gathering and decisionmaking processes involved in managing the diagnosis of carpal tunnel syndrome (CTS). Many Americans experience symptoms of CTS and expect relief from the condition, which can be accomplished only with proper diagnosis. If the CTS is diagnosed early, we expect better results of treatment.

For the purpose of this guideline, CTS is defined as a symptomatic compression neuropathy of the median nerve at the level of the wrist, characterized physiologically by evidence of increased pressure within the carpal tunnel and decreased function of the nerve at that level. CTS can be caused by many different diseases, conditions and events. It is characterized by patients as producing numbness, tingling, hand and arm pain and muscle dysfunction. The disorder is not restricted by age, sex, ethnicity, or occupation and is associated with or caused by systemic disease and local mechanical and disease factors.

The AAOS clinical practice guidelines are developed using evidence-based methods, and the recommendations in them are based on systematic reviews of the available literature. The purpose of systematically performing a review is to combat bias. Substantial documentation accompanies the guideline to ensure that the recommendations are, indeed, unbiased. Ideally, those who wish to perform an "intellectual audit" of a guideline can examine this documentation and independently arrive at the same recommendations.

Because systematic reviews combat bias, the studies included in them are not chosen on the basis of whether they were published by an expert. Similarly, the physician work group members who prepared this guideline did not begin work on it by exchanging articles from 
their personal files. Rather, articles were identified using comprehensive searches of several electronic databases and were included in the guideline only when they met specific criteria that were developed before work on the guideline began.

To further combat bias, the information extracted from published articles did not include the conclusions of the articles' authors (who, themselves, might be biased). Rather, the focus of the guideline and the systematic review upon which it is based is on the data and how they were collected. Thus, information for the guideline was principally derived from information contained in an article's Methods and Results sections. A total of 224 full articles were reviewed for this guideline, 24 of which were ultimately concluded.

These systematic reviews were conducted between August 2006 and March 2007. They identify good evidence as well as circumstances in which evidence is lacking. They also identify topics for future research to improve the treatment of patients with osteoarthritis of the knee. AAOS staff and the Carpal Tunnel Syndrome Guideline Work Group systematically reviewed the available literature and subsequently wrote the following recommendations based on a rigorous, standardized process.

Musculoskeletal care is provided in many different settings by many different providers. We created this guideline as an educational tool to guide qualified physicians through a series of treatment decisions in an effort to improve the quality and efficiency of care. This guideline should not be construed as including all proper methods of care or excluding methods of care reasonably directed to obtaining the same results. The ultimate judgment regarding any specific procedure or treatment must be made in light of all circumstances presented by the patient and the needs and resources particular to the locality or institution.

\section{Methods}

The methods used to develop this clinical practice guideline were designed to combat bias, enhance transparency, and promote reproducibility. Their purpose is to allow interested readers the ability to inspect all of the information the work group used to reach all of its decisions and to verify that these decisions are in accord with the best available evidence. The draft of this guideline was subject to peer review and public commentary, and it was approved by the AAOS Evidence-Based Practice Committee; Guidelines and Technology Committee; Council on Research, Quality Assessment and Technology; and the Board of Directors. The methods used to prepare this guideline are detailed in the full clinical practice guideline, which is available at http://www.aaos.org/research/guidelines/ CTS_guideline.pdf. ${ }^{1}$

\section{Grading the Recommendations}

Full interpretation of the recommendations in this guideline depends on understanding the grade assigned to each recommendation. These grades are the following:

A Good evidence (level I studies with consistent findings) for or against recommending intervention. 
B Fair evidence (level II or III studies with consistent findings) for or against recommending intervention.

C Poor-quality evidence (level IV or V) for or against recommending intervention.

I There is insufficient or conflicting evidence not allowing a recommendation for or against intervention.

\section{Recommendations}

\section{Recommendation 1: History of Present Illness}

Recommendation 1.1-The physician should obtain an accurate patient history. Several broad questions may solicit reports of hand numbness or hand/wrist pain and/or symptoms. These include

a.

b.

c.

d.

e.

f.
Duration: How long have the symptoms been present?

Severity/character: How severe are the symptoms? When do symptoms occur (eg, nighttime)? Describe symptom quality (eg, tingling, burning, aching). Is the numbness/tingling intermittent or persistent? What improves (eg, shaking the hand, holding hand down, warm water) or exacerbates (eg, driving, holding a telephone, using vibratory tools) symptoms?

Location/radiation: What is the location of the pain and/or numbness? Are the symptoms in the median nerve distribution of the hand(s)? If the symptom is not focal, does it radiate to a specific area of the body? Are there any other associated symptoms (eg, numbness in the feet; symptoms in the neck, shoulder, and more proximal in the arm; weakness, clumsiness, dropping things)? Pace of illness: Is the problem getting better, worse, or staying the same? If it is changing, what has been the rate of change?

Previous treatment: What has been done and what makes it better (eg, splint, injections, therapy, activity modification, medications)?

Lifestyle and activities: What hand activities are common for the patient (eg, hobbies, occupation)? Are there functional limitations due to these symptoms?

Grade of Recommendation: C

Obtaining a history relating to CTS serves to assist in diagnosing the disease and planning appropriate treatment options. A high level of evidence is not available in the literature to assess the diagnostic utility of history data and the predictive value of the data in evaluating severity of disease and outcomes of treatment. Therefore, the work group employed expert opinion in creating this recommendation to guide physicians in the appropriate questions that should be asked during patient encounters to improve diagnosis. 
It is unclear from the literature whether the duration of symptoms correlates with the amount of nerve injury or can predict treatment outcomes. It is also unclear whether the severity and character of symptoms are related to the structural and physiologic condition of the median nerve. The evidence from available studies does not illustrate a direct relationship between symptoms and electrodiagnostic test results either across all studies (regardless of design) or by looking at results for a single electrodiagnostic test. Symptoms such as numbness along the distribution of the median nerve, increasing symptoms at night when sleeping, improvement of symptoms by shaking the hand, and symptom exacerbation when driving or holding a telephone are classic symptoms that in combination may be highly suggestive of CTS.

Identification of the location and radiation of symptoms may exclude or include other diagnoses, such as proximal nerve compression syndrome, that may mimic CTS. Having these associated symptoms may alert the physician to look for diagnoses other than CTS.

The symptoms of CTS may vary on a daily basis. Understanding the disease progression can be helpful in determining the appropriate diagnosis and treatment plan. For symptoms that are not increasingly severe but vary periodically, conservative management may be helpful in diagnosis.

Understanding patient response to previous treatment may verify the diagnosis of CTS and may support patient progression to carpal tunnel surgery. Response to conservative treatment varies. If the physician uses steroid injection, the greatest patient improvement is seen at 1 month. If the physician uses conservative management and measures improvement with electrodiagnostic tests, the greatest improvement is between 3 and 6 months, as seen by change in distal motor latency measurements (ms) (Figure 102 in the Clinical Guideline ${ }^{2}$ ).

In summary, the work group used expert opinion to formulate Recommendation 1 for obtaining appropriate history to make the diagnosis of CTS. Based on the level of evidence for the studies that reviewed conservative management, the work group rates this recommendation at a $\mathrm{C}$ level, which indicates poor quality of evidence consisting of level II, III, or IV data for or against recommending intervention (Table $12^{3}$ and Figure $8^{2}$ in the Clinical Guideline).

\section{Recommendation 2: Physical Examination}

The physician should perform a physical examination of the patient that may include
a. Personal characteristics (eg, age, sex, weight, height, body habitus)
b. Range of motion of hand/wrist
c. Observation of deformity, swelling, atrophy, skin trophic changes
d. Pinch/grip strength
e. Hand diagram
f. Sensory examination (eg, two-point discrimination, Semmes-Weinstein monofilament, vibrometry, texture discrimination) 
g. Manual muscle testing of the upper extremity (eg, examine for muscular atrophy, especially in the thenar muscle group)

h. Provocative tests (eg, Phalen test, Tinel sign, median nerve compression test, reverse Phalen test)

i. Discriminatory examination for alternative diagnoses (eg, radiculopathy, neuropathy, pain syndromes, arthritis, tendinitis, vascular abnormalities)

Level of Evidence: $\mathrm{V}$

Grade of Recommendation: C

Personal characteristics such as age, sex, height, weight, and body habitus are basic information that are important for fulfilling the evaluation and management documentation. Included in parts of this evaluation would be determination of vascular status, obvious deformity, and testing for other related conditions (eg, basilar thumb arthritis, de Quervain disease, cubital tunnel syndrome, cervical radiculopathy).

Sensory examination using, for example, two-point discrimination, Semmes-Weinstein monofilament, vibrometry, and texture discrimination, is important for defining the anatomic distribution of sensory changes. Sufficient evidence does not exist from the literature reviewed for this guideline to recommend one test over another or to suggest the overall utility of a test in diagnosing CTS. Future research will be required to confirm the diagnostic utility of these tests.

Manual muscle testing of the upper extremity, including evaluation for obvious muscle atrophy, is important, especially for the thenar muscle area. Thenar atrophy has been reported to have a high predictive value in CTS, but its appearance can be rare. ${ }^{4}$ This literature review contained only two primary studies ${ }^{4,5}$ that specifically addressed thenar atrophy. No conclusion on the diagnostic value of thenar atrophy can be drawn conclusively from two studies.

Several provocative tests should be considered to aid in the evaluation and diagnosis of CTS. For multiple reasons (ie, poor study design, variability in study results, inconsistent data, few studies examining the same tests, small datasets for each test), no one test has been identified as a gold standard for identifying CTS. Phalen test results ranged in sensitivity from 0.46 to 0.80 and in specificity from 0.51 to $0.91 .^{5-8}$ The Tinel sign ranged in sensitivity from 0.28 to 0.73 and in specificity from 0.44 to $0.95 .^{4-7}$ The median nerve compression test ranged in sensitivity from 0.04 to 0.79 and in specificity from 0.25 to $0.96 .{ }^{5,8,9} \mathrm{Combining}$ the results of more than one provocative test might increase the sensitivities and specificities. For example, combined results of the Phalen and median nerve compression tests yielded a sensitivity of 0.92 and a specificity of $0.92 ;{ }^{8}$ however, further literature would be required to confirm whether this result is diagnostically accurate.

The reverse Phalen test, tethered median nerve stress test, and the tourniquet test have been evaluated only a few times, leaving insufficient evidence from which to draw any conclusions as to their accuracy in the diagnosis of CTS. ${ }^{4-6}$ 
The clinical tests for CTS by themselves do not reliably diagnose CTS. Similarly, as will be discussed in Recommendation 3, electrodiagnostic studies by themselves also do not reliably diagnose CTS. However, when the symptoms, clinical tests, and electrodiagnostic tests are combined, statistical significance $(P<0.05)$ is obtained compared with postsurgical outcomes. Among surgical candidates, clinical tests and electrodiagnostic studies correlated with positive postsurgical outcomes (Figures 96 through 99 in the Clinical Guideline ${ }^{2}$ ).

Although CTS cannot be diagnosed primarily on clinical or electrodiagnostic grounds, the evidence shows that a combination of the two can better confirm the diagnosis and provide treatment orientation vis-à-vis carpal tunnel release. These combination tests vary considerably among the studies. For example:

Haupt et al: ${ }^{10}$ (motor and sensory tests) + (distal motor latency of the median nerve, antidromic sensory nerve conduction velocity [NCV], electromyography [EMG] examination of abductor pollicis brevis)

Braun and Jackson: ${ }^{11}$ (range of motion, grip strength, pinch strength, monofilament sensory evaluation, Phalen, Tinel) + (sensory latency $>3.5 \mathrm{~ms}$ )

Glowacki et al: ${ }^{12}$ (Phalen or Tinel) + (motor latencies $>4.0 \mathrm{~ms}$, sensory latencies $>3.7 \mathrm{~ms}$, amplitudes $<20 \mu \mathrm{V}$, or a conduction velocity $<50 \mathrm{~m} / \mathrm{s}$ with evidence of fibrillation)

Boniface et al: ${ }^{13}$ (sensory tests) + (prolonged median sensory conduction velocity, distal motor latency to abductor pollicis brevis)

Given the small number of studies in the diagnostic groupings illustrated in these examples, definitive combinations of clinical and electrodiagnostic tests that provide the best performance could not be identified. Future research should compare the diagnostic accuracy of different combinations of tests to identify and recommend specific test combinations.

Other conditions may present with symptoms similar to those found in CTS (eg, cervical radiculopathy, hypothyroidism, peripheral neuropathy, wrist/trapeziometacarpal arthrosis, wrist tendinitis/tenosynovitis, Raynaud disease, arterial injury or thrombosis, nerve laceration, neuroma, brachial plexus injury, other nerve entrapment syndromes, pain syndromes). Physical examination should include other areas of the upper extremity and neck that may relate to these alternative diagnoses. This may include the following:

1. Muscular atrophy or weakness of the shoulder, elbow, wrist, thumb and/or fingers

2. Active range of motion, particularly for the wrist and digits

3. Swelling, masses, and/or tenderness around the wrist or digits

4. Capillary refill, radial and ulnar pulses, Allen test

5. Wounds or scars on the upper extremity

6. Cervical spine range of motion, Spurling sign 
7. Thyroid enlargement

8. Radiographs of the wrist

These recommendations were formed by expert opinion of the work group; no literature searches were performed to support these recommendations.

\section{Recommendation 3: Nerve Conduction Velocity Studies}

Recommendation 3.1a-The physician may obtain electrodiagnostic tests to differentiate among diagnoses.

Recommendation 3.1b-The physician may obtain electrodiagnostic tests in the presence of thenar atrophy and/or persistent numbness.

Level of Evidence: V

Grade of Recommendation: C

There were no prospective studies that evaluated a broad spectrum of patients. In addition, there was no consistent independent reference standard (including atrophy and persistent numbness) used across studies, and none attempted to differentiate CTS from other diagnoses. There were multiple studies differentiating CTS patients from normal subjects, mainly as case-control or cohort studies (Figures 5 and 9 in the Clinical Guideline ${ }^{2}$ ). There were considerable problems with spectrum bias and lack of a gold standard for diagnosis. Thus, the quality of studies fell to level V, or expert opinion. Despite this, there was expert opinion that, in some cases, NCV studies and needle EMG can differentiate CTS from other peripheral nerve problems (eg, polyneuropathy, brachial plexopathy, cervical radiculopathy). Additionally, thenar atrophy and/or persistent numbness may suggest severe nerve injury, which may warrant more aggressive management or diagnostic evaluation. NCV study and EMG can assist in documenting this severe nerve injury.

Recommendation 3.1c-The physician should obtain electrodiagnostic tests if clinical and/or provocative tests are positive and surgical management is being considered.

Levels of Evidence: II and III

Grade of Recommendation: B

Improvement in symptoms after surgical release is a clinically relevant reference standard that is important to patient care and patient management. Four studies ${ }^{11-14}$ found a correlation between a combination of clinical presentation (symptoms and Phalen test or Tinel sign) and nerve conduction studies with good surgical outcome (Figure 98 in the Clinical Guideline $^{2}$ ). See the meta-regression of surgical outcome and clinical and electrodiagnostic tests (Figure 99 in the Clinical Guideline ${ }^{2}$ ). The association with clinical tests alone or electrodiagnostic tests alone did not have a statistically significant association with surgical outcome (Figures 96 and 97 in the Clinical Guideline ${ }^{2}$ ). There were no other high-quality studies that attempted to correlate outcomes of other treatments (eg, splinting, steroid injections) with pretreatment diagnostic results. Good response to surgery does not prove the diagnosis of CTS because other factors may provide symptom relief (eg, placebo, surgery activity modification, postoperative splinting). However, postsurgical improvement 
is a clinically relevant outcome. Clinicians who are considering surgical management should know that good surgical outcome is correlated with a combination of positive clinical and positive electrodiagnostic tests. ${ }^{10-14}$ Again, it is important to note that the evidence shows a statistically significant association between the combination of clinical tests, electrodiagnostic testing, and positive surgical outcomes (Figures 96 through 99 in the Clinical Guideline ${ }^{2}$.

The literature does not permit calculation of sensitivities and specificities of all tests. Additionally, sensitivity and specificity of NCV studies cannot be determined with absolute certainty primarily because a diagnosis of CTS cannot be made with absolute certainty without a gold standard. The lack of a reliable gold standard and the use of reference standards that are variable from study to study give disparate results. Further, no universally agreed-upon diagnostic criteria exist for the diagnosis of CTS. The case-control studies for electrodiagnostic testing consistently showed receiver operating curves of a very high specificity and variable sensitivity (Figure 11 in the Clinical Guideline ${ }^{2}$ ), illustrating inflated results due to spectrum bias. The case-control studies are, therefore, not considered useful.

Few well-designed cross-sectional and cohort studies were found. Based on the low availability of quality studies as well as the small size of the study populations, statistically significant receiver operating curves ${ }^{1}$ could not be constructed.

Recommendation 3.2-If the physician orders electrodiagnostic tests, the testing protocol should follow the American Academy of Neurology/American Association of Neuromuscular and Electrodiagnostic Medicine/American Academy of Physical Medicine and Rehabilitation (AAN/AANEM/AAPMR) guidelines for diagnosis of CTS.

a. Sensory NCV studies to the median nerve with distal latency compared to the ulnar and radial nerve

b. Median motor nerve conduction in most patients

c. Needle EMG at the discretion of the physician

Levels of Evidence: IV and V

Grade of Recommendation: C

Thirteen published studies were evaluated and met criteria for review compared with a reference standard of signs, symptoms, and one electrodiagnostic test. Twelve of these studies ${ }^{15-26}$ were level IV and one ${ }^{27}$ was level II. The reference standard used included signs, symptoms, and a different electrodiagnostic test than the electrodiagnostic test being evaluated in the study. The index test could not be part of the referenced standard, and vice versa. These inclusion criteria were comparable but slightly different from the criteria used in the AANEM Practice Parameter, which the work group agreed to use as a protocol for electrodiagnostic testing. The AANEM reference standard included clinical symptoms and did not include another nerve conduction study. ${ }^{28}$

The vast majority of studies were of case-control design, suggesting spectrum bias. A result of spectrum bias is that the sensitivities and specificities are likely inflated. Multiple nerve 
conduction studies were reported in the primary articles. These articles employed 11 different nerve conduction study techniques (Figure 6 in the Clinical Guideline ${ }^{2}$ ), including median sensory to ulnar sensory comparison, median sensory to radial sensory comparison, mixed nerve comparison, and median motor testing. A review of these studies again could not determine conclusively that any of the electrodiagnostic tests was clearly superior (Table 3 in the Clinical Guideline ${ }^{3}$ ). Comparisons cannot be made, and a test hierarchy cannot be constructed, ${ }^{1}$ when case-control studies are used because of the probability of spectrum bias. The authors of Diagnosis and Treatment of Worker-Related Musculoskeletal Disorders of the Upper Extremity, ${ }^{29}$ another evidence-based systematic review that examined 31 databases and 1,600 journals, found similar conclusions.

The 2002 publication of the AANEM, AAN, and AAPMR, whose protocol is preferred by the work group for electrodiagnostic testing, was an evidence-based review of 278 articles, of which 22 met literature inclusion criteria. This review also illustrated that there is no conclusive evidence that one single study has the highest sensitivity or specificity. It did, however, conclude that median sensory latencies across the wrist compared to ulnar or radial latencies or proximal median conduction improved sensitivity as opposed to absolute median sensory or motor distal latencies. ${ }^{28}$

Recommendation 3.3-The physician should not routinely evaluate patients suspected of having CTS with new technology such as magnetic resonance imaging (MRI), CT, and pressure-specified sensorimotor devices (PSSDs).

\section{Level of Evidence: V}

Grade of Recommendation: C

Changes in the appearance of the median nerve have been reported with MRI and CT. Following the face-to-face work group meeting on February 24, 2007, an additional nonsystematic literature review was completed. This review included 6 of 25 articles on MRI and no studies on CT. Five of the six articles on MRI were case-control study designs and therefore introduced spectrum bias. There is no good evidence to date that these imaging modalities improve sensitivity or specificity in making the diagnosis of CTS ${ }^{29}$ In the opinion of this committee, there is no role for the routine use of MRI or CT in the diagnosis or management of CTS. These imaging modalities should be reserved for the evaluation of related conditions in the appropriate clinical setting (eg, Kienbock disease, ruling out occult fracture, hook of hamate nonunion).

PSSDs have also been described for evaluation of patients with symptoms of CTS. Two of three studies investigating this technology were included in this additional literature review process. Two studies do not provide sufficient evidence to provide a recommendation. There is, however, significant experience and literature supporting the use of NCV/EMG in the evaluation of CTS. In the opinion of this committee, there is insufficient evidence to support the routine use of PSSDs in the evaluation of CTS at this time. 


\section{Future Research}

Although every effort has been made to include studies of the highest evidence, high-quality evidence is not readily available for CTS diagnosis at this time. This guideline has been hindered by a relative lack of studies of the best design (ie, level I and II evidence) and specifically hindered by an abundance of case-control studies (which are subject to spectrum bias). To achieve a high-quality literature base, academic authors and scientists should invest their time and effort in studies designed to avoid bias (eg, blinded controlled trials that are not case-control studies). Future studies should, from the onset, be based on improved study design. The recommendations of this guideline have therefore depended to some degree on lesser evidence, including consensus and expert opinion. Few studies describe the impact of health policy on process optimization. Skillful and timely delivery of CTS care, the access that patients have to definitive management, and the choices patients make in the management of disease all affect outcomes. Studies of cost burden to the community from a common disabling source of productivity loss need restatement, and future studies must address all of these issues.

Although thousands of publications have been written regarding the diagnosis and treatment of CTS, greater precision and transparent methodology are needed to bring the next generation of academic papers to the forefront of review so that they directly contribute to quality medical care. The process of periodically assessing our medical and surgical progress in diagnosing and treating common conditions becomes more precise each year. A common protocol for evaluation of the literature should spur more consistently sound design and greater health for the cost of care expended. This clinical practice guideline is based on stronger evidence-based analysis methodology than ever previously conducted by the AAOS and is therefore state-of-the-art. The details of the statistical methods used in this guideline are further explained at http://www.aaos.org/Research/guidelines/CTSdiagnosisguide.asp. ${ }^{30}$

\section{Acknowledgments}

Dr. Masear or a member of her immediate family serves as a board member, owner, officer, or committee member of the American Society for Surgery of the Hand and is affiliated with the publication The American Journal of Orthopedics. Dr. Chung or a member of his immediate family has received research or institutional support from Stryker. Dr. Andary or a member of his immediate family is a member of a speakers' bureau or has made paid presentations on behalf of Pfizer and Allergan. Dr. Amadio or a member of his immediate family serves as a board member, owner, officer, or committee member of the Orthopaedic Research Society, and Immanuel St. Joseph Hospital; is affiliated with the publication/publishers Journal of Orthopaedic Research and Saunders/MosbyElsevier; has received research or institutional support from Musculoskeletal Transplant Foundation and the National Institutes of Health (NIAMS and NICHD); has stock or stock options held in Johnson \& Johnson, Merck, and Procter \& Gamble; and has received nonincome support (such as equipment or services), commercially derived honoraria, or other non-research-related funding (such as paid travel) from the Journal of Bone and Joint Surgery American. Dr. Barth or a member of his immediate family has stock or stock options held in Amgen, Merck, and Pfizer. Dr. Watters or a member of his immediate family serves as a board member, owner, officer, or committee member of Bone and Joint Decade USA, North American Spine Society, Intrinsic Therapeutics, Work Loss Data Institute, and American Board of Spine Surgery; is affiliated with the publication The Spine Journal; serves as a paid consultant to or is an employee of Blackstone Medical, Medtronic Sofamor Danek, Stryker, Intrinsic Therapeutics, and McKessen Health Care Solutions; and has stock or stock options held in Intrinsic Therapeutics. Dr. Goldberg is affiliated with the Journal of Pediatric Orthopedics and the Journal of Children's Orthopaedics. Dr. Haralson or a member of his immediate family serves as a paid consultant to or is an employee of Medtronic and Medtronic Sofamor Danek and has stock or stock options held in Orthofix. Ms. Wies or a member of her immediate family has stock or stock options held in Shering Plough. None of the following authors or a member of their immediate families has received anything of value from or owns stock in a commercial company or institution related directly or indirectly to the subject of this article: Dr. Keith, Dr. Maupin, and Dr. Turkelson. 


\section{References}

Please note that reference 28 has been withdrawn by the publisher. This does not compromise the integrity of the AAOS Clinical Practice Guideline on the Diagnosis of Carpal Tunnel Syndrome.

1. American Academy of Orthopaedic Surgeons. Clinical Guideline on Diagnosis of Carpal Tunnel Syndrome. Rosemont, IL: American Academy of Orthopaedic Surgeons; 2007 May. Available at: http://www.aaos.org/research/guidelines/CTS_guideline.pdf [Accessed March 11, 2009]

2. American Academy of Orthopaedic Surgeons. Clinical Guideline on Diagnosis of Carpal Tunnel Syndrome. Figures. Rosemont, IL: American Academy of Orthopaedic Surgeons; 2007 May. Available at: http://www.aaos.org/research/guidelines/CTS_figs.pdf [Accessed March 11, 2009]

3. American Academy of Orthopaedic Surgeons. Clinical Guideline on Diagnosis of Carpal Tunnel Syndrome. Evidence Tables. Rosemont, IL: American Academy of Orthopaedic Surgeons; 2007 May. Available at: http://www.aaos.org/research/guidelines/CTS_tables.pdf [Accessed March 11, 2009]

4. Gomes I, Becker J, Ehlers JA, Nora DB. Prediction of the neurophysiological diagnosis of carpal tunnel syndrome from the demographic and clinical data. Clin Neurophysiol. 2006; 117:964-971. [PubMed: 16516550]

5. de Krom MC, Knipschild PG, Kester AD, Spaans F. Efficacy of provocative tests for diagnosis of carpal tunnel syndrome. Lancet. 1990; 335:393-395. [PubMed: 1968125]

6. Raudino F. Tethered median nerve stress test in the diagnosis of carpal tunnel syndrome. Electromyogr Clin Neurophysiol. 2000; 40:57-60. [PubMed: 10782359]

7. Katz JN, Larson MG, Sabra A, et al. The carpal tunnel syndrome: Diagnostic utility of the history and physical examination findings. Ann Intern Med. 1990; 112:321-327. [PubMed: 2306060]

8. Fertl E, Wober C, Zeitlhofer J. The serial use of two provocative tests in the clinical diagnosis of carpal tunnel syndrome. Acta Neurol Scand. 1998; 98:328-332. [PubMed: 9858103]

9. Kaul MP, Pagel KJ, Wheatley MJ, Dryden JD. Carpal compression test and pressure provocative test in veterans with median-distribution paresthesias. Muscle Nerve. 2001; 24:107-111. [PubMed: 11150972]

10. Haupt WF, Wintzer G, Schop A, Lottgen J, Pawlik G. Long-term results of carpal tunnel decompression: Assessment of 60 cases. J Hand Surg [Br]. 1993; 18:471-474.

11. Braun RM, Jackson WJ. Electrical studies as a prognostic factor in the surgical treatment of carpal tunnel syndrome. J Hand Surg [Am]. 1994; 19:893-900.

12. Glowacki KA, Breen CJ, Sachar K, Weiss AP. Electrodiagnostic testing and carpal tunnel release outcome. J Hand Surg [Am]. 1996; 21:117-121.

13. Boniface SJ, Morris I, Macleod A. How does neurophysiological assessment influence the management and outcome of patients with carpal tunnel syndrome? Br J Rheumatol. 1994; 33:1169-1170. [PubMed: 8000749]

14. Prick JJ, Blaauw G, Vredeveld JW, Oosterloo SJ. Results of carpal tunnel release. Eur J Neurol. 2003; 10:733-736. [PubMed: 14641522]

15. Sheu JJ, Yuan RY, Chiou HY, Hu CJ, Chen WT. Segmental study of the median nerve versus comparative tests in the diagnosis of mild carpal tunnel syndrome. Clin Neurophysiol. 2006; 117:1249-1255. [PubMed: 16600674]

16. Lew HL, Date ES, Pan SS, Wu P, Ware PF, Kingery WS. Sensitivity, specificity, and variability of nerve conduction velocity measurements in carpal tunnel syndrome. Arch Phys Med Rehabil. 2005; 86:12-16. [PubMed: 15640982]

17. Sharma KR, Rotta F, Romano J, Ayyar DR. Early diagnosis of carpal tunnel syndrome: Comparison of digit 1 with wrist and distoproximal ratio. Neurol Clin Neurophysiol. 2001; 2001(2):2-10.

18. Cherniack MG, Moalli D, Viscolli C. A comparison of traditional electrodiagnostic studies, electroneurometry, and vibrometry in the diagnosis of carpal tunnel syndrome. J Hand Surg [Am]. 1996; 21:122-131. 
19. Foresti C, Quadri S, Rasella M, Tironi F, Viscardi M, Ubiali E. Carpal tunnel syndrome: Which electrodiagnostic path should we follow? A prospective study of 100 consecutive patients. Electromyogr Clin Neurophysiol. 1996; 36:377-384. [PubMed: 8891478]

20. Kothari MJ, Rutkove SB, Caress JB, Hinchey J, Logigian EL, Preston DC. Comparison of digital sensory studies in patients with carpal tunnel syndrome. Muscle Nerve. 1995; 18:1272-1276. [PubMed: 7565924]

21. Kuntzer T. Carpal tunnel syndrome in 100 patients: Sensitivity, specificity of multineurophysiological procedures and estimation of axonal loss of motor, sensory and sympathetic median nerve fibers. J Neurol Sci. 1994; 127:221-229. [PubMed: 7707081]

22. Uncini A, Di Muzio A, Cutarella R, Awad J, Gambi D. Orthodromic median and ulnar fourth digit sensory conductions in mild carpal tunnel syndrome. Neurophysiol Clin. 1990; 20:53-61. [PubMed: 2348811]

23. Jackson DA, Clifford JC. Electrodiagnosis of mild carpal tunnel syndrome. Arch Phys Med Rehabil. 1989; 70:199-204. [PubMed: 2923541]

24. Seror P. The value of special motor and sensory tests for the diagnosis of benign and minor median nerve lesion at the wrist. Am J Phys Med Rehabil. 1995; 74:124-129. [PubMed: 7710726]

25. Lesser EA, Venkatesh S, Preston DC, Logigian EL. Stimulation distal to the lesion in patients with carpal tunnel syndrome. Muscle Nerve. 1995; 18:503-507. [PubMed: 7739637]

26. Sander HW, Quinto C, Saadeh PB, Chokroverty S. Sensitive median-ulnar motor comparative techniques in carpal tunnel syndrome. Muscle Nerve. 1999; 22:88-98. [PubMed: 9883861]

27. Schuhfried O, Vacariu G, Kopf A, Paternostro-Sluga T. Relative slowing of the median antidromic sensory nerve conduction velocity to the ring finger in screening for carpal tunnel syndrome. $\mathrm{J}$ Hand Surg [Am]. 2004; 29:947-952.

28. Jablecki CK, Andary MT, Floeter MK, et al. Second AAEM literature review of the usefulness of nerve conduction studies and needle electromyography for the evaluation of patients with carpal tunnel syndrome. Muscle Nerve Suppl. 2002; 25:918-922.

29. Chapell, R.; Turkelson, CM.; Coates, V., et al. Diagnosis and Treatment of Worker-Related Musculoskeletal Disorders of the Upper Extremity: Evidence Report/Technology Assessment Number 62. Rockville, MD: Agency for Healthcare Research and Quality.; 2002 Dec. (Prepared by ECRI, Health Technology Assessment Group under Contract No. 290-97-0020.) AHRQ Publication No. 02-E038

30. American Academy of Orthopaedic Surgeons. Clinical Guideline on Diagnosis of Carpal Tunnel Syndrome. Rosemont, IL: American Academy of Orthopaedic Surgeons; 2007 May. Available at: http://www.aaos.org/research/guidelines/CTSdiagnosisguide.asp [Accessed March 11, 2009] 